\title{
ON COHOMOLOGY GROUPS OF CERTAIN SUBCOMPLEXES OF DOLBEAULT COMPLEXES ${ }^{1}$
}

\author{
SHAW MONG
}

\begin{abstract}
The paper first shows that for a path connected topological group acting analytically on a complex manifold $M$, the induced action on the cohomology groups of antiholomorphic forms on $M$ is trivial. By the same method it then shows that the cohomology groups of complex $\left\{\operatorname{ker} \partial^{p, *}\right\}$ can be injected into the Dolbeault cohomology groups.
\end{abstract}

1. Introduction. For a smooth $G$-manifold with $G$ a path connected topological group, it is well known that the induced $G$-action on the DeRham cohomology groups is trivial. On the other hand the action on the Dolbeault cohomology groups may fail to be trivial. In this paper we show that the action is trivial on its subcomplex $\left\{\Gamma_{a}\left(\Lambda^{0, *}\right)\right\}$ of antiholomorphic forms. In particular we prove:

(1.1) THEOREM. Let $G$ be a path connected topological group acting analytically on a complex manifold $M$ (of $m$ complex dimensions). Then the induced $G$-actions on the cohomology groups of

$$
\begin{aligned}
0 \longrightarrow \Gamma_{a}\left(\Lambda^{0,0}(M)\right) & \longrightarrow \cdots \longrightarrow \Gamma_{a}\left(\Lambda^{0, q}(M)\right) \\
& \stackrel{\delta}{\longrightarrow} \Gamma_{a}\left(\Lambda^{0, q+1}(M)\right) \longrightarrow \cdots \longrightarrow \Gamma_{a}\left(\Lambda^{0, m}(M)\right) \longrightarrow 0
\end{aligned}
$$

are trivial, where $\Gamma_{a}\left(\Lambda^{0 . q}(M)\right)$ is the complex vector space of antiholomorphic $(0, q)$ forms on $M$.

The proof, similar to that of the Poincaré Lemma, appears in $\S 3$. We also prove:

(1.2) THEOREM. Let $M$ be a complex manifold (of $m$ complex dimensions) which can be deformed analytically onto a point in $M$ and let $\operatorname{ker} \partial^{p, q}$

Received by the editors May 30, 1972 and, in revised form, September 19, 1972.

AMS (MOS) subject classifications (1970). Primary 58A10, 58G05.

Key words and phrases. G-manifolds, differential forms, antiholomorphic forms, DeRham complexes, Dolbeault complexes, cohomology groups.

${ }^{1}$ This paper is a portion of the author's doctoral thesis written under the direction of Professor Joseph Sampson at the Johns Hopkins University. Research supported by National Science Foundation Grant GU-3171.

(c) American Mathematical Society 1973 
denote the kernel of $\partial$ on the space, $\Gamma\left(\Lambda^{p, q}(M)\right)$, of differential $(p, q)$ forms; then for $0 \leqq p \leqq m$, the following inclusions $i$ :

$0 \rightarrow \operatorname{ker} \partial^{p, 0} \stackrel{\delta}{\rightarrow} \cdots \stackrel{\delta}{\rightarrow}$ ker $\partial^{p, q} \stackrel{\delta}{\rightarrow}$ ker $\partial^{p, q+1} \stackrel{\delta}{\rightarrow} \cdots \stackrel{\delta}{\rightarrow}$ ker $\partial^{p, m} \rightarrow 0$

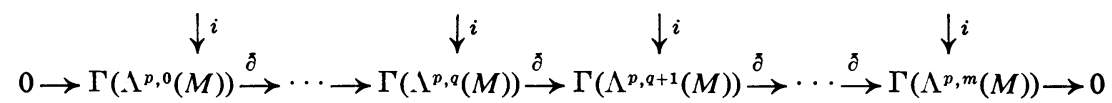

induce injections:

$$
i^{*}: H^{q}\left(\operatorname{ker} \partial^{p, *}\right) \rightarrow H^{q}\left(\Lambda^{p, *}(M)\right), \text { for all }(p, q) \neq(0,0) .
$$

Applying to the polydisc $\Delta$ in $C^{m}$ and observing that $H^{q}\left(\Lambda^{p, *}(\Delta)\right)=0$ for all $q \geqq 1$, we have a result similar to a result in [1]:

(1.3) Corollary. For all $0 \leqq p \leqq m$, the complexes

$\operatorname{ker} \partial^{p, 0} \stackrel{\delta}{\longrightarrow} \cdots \stackrel{\delta}{\longrightarrow} \operatorname{ker} \partial^{p, q} \stackrel{\delta}{\longrightarrow} \operatorname{ker} \partial^{p, q+1} \stackrel{\delta}{\longrightarrow} \cdots \stackrel{\delta}{\longrightarrow} \operatorname{ker} \partial^{p, m} \longrightarrow 0$ are exact over the polydisc $\Delta$ in $C^{m}$.

The author would like to express his gratitude to Professor Sampson for suggestions which brought the original boring mechanical computations into the present form.

2. Preliminary notations. Throughout this paper, except as noted, all differential forms are expressed and computed in global forms.

Two holomorphic mappings $g^{0}$ and $g^{1}$ on the complex manifold $M$ are said to be analytically homotopic, if they are homotopic through a family of holomorphic mappings on $M$. Let $g(z, t): M \times I \rightarrow M$ be such a homotopy linking $g^{0}$ and $g^{1}$; we shall use notation $g^{t}(z)$ to stress the holomorphic mapping $g(z, t)$ with $t$ fixed.

After identifying the cotangent bundle $T^{*}(M \times I)$ of $M \times I$ with $T^{*} M \oplus$ $T^{*} I$, we can write $d$, the exterior derivative in the DeRham complex of $M \times I$, as $\partial+\bar{\partial}+d_{t}$, where $d_{t}$ is the exterior derivative with respect to the variable $t$. With this understanding, a differential form on $M \times I$ is called a $(p, q, 0)$ or $(p, q, 1)$ form, according to whether it is of type

or of type

$$
\sum_{|I|=p,|J|=q} \omega_{I, J}(z, t) d z_{I} \wedge d \bar{z}_{J}
$$

$$
\sum_{|I|=p,|J|=q} \omega_{I, J}(z, t) d z_{I} \wedge d \bar{z}_{J} \wedge d t
$$

resp. Obviously any differential form of $M \times I$ is locally a linear combination of such forms.

An analytic homotopy $g(z, t): M \times I \rightarrow M$ induces a sequence of linear mappings $g^{*}: \Gamma\left(\Lambda^{*}(M)\right) \rightarrow \Gamma\left(\Lambda^{*}(M \times I)\right)$, which has the following properties. 
First, for any differential form $\omega$ on $M$,

$$
g^{*}(\partial+\bar{\partial}) \omega=\left(\partial+\bar{\partial}+d_{t}\right) g^{*} \omega
$$

For any $(p, q)$ form $\omega$,

$$
g^{*} \omega=\left(\omega^{\prime}+\omega^{\prime \prime}+\omega^{\prime \prime \prime}\right) \wedge d t
$$

where $\omega^{\prime}, \omega^{\prime \prime}, \omega^{\prime \prime \prime}$ are $(p, q, 0),(p, q-1,0),(p-1, q, 0)$ forms resp. Notice also that

$$
\left(g^{t *} \omega\right)(z)=\omega^{\prime}(z, t)
$$

Suppose that $\partial \omega=0$, then

$$
\begin{aligned}
g^{*} \bar{\partial} \omega & =g^{*}(\bar{\partial}+\partial) \omega=\left(\bar{\partial}+\partial+d_{t}\right) g^{*} \omega \\
& =\left(\bar{\partial}+\partial+d_{t}\right)\left(\omega^{\prime}+\left(\omega^{\prime \prime}+\omega^{\prime \prime \prime}\right) \wedge d t\right) .
\end{aligned}
$$

Observe that the $(p, q, 1)$ form on the r.h.s. is

$$
\left(\partial \omega^{\prime \prime \prime}+\bar{\partial} \omega^{\prime \prime}+(-1)^{p+q} \frac{d \omega^{\prime}}{d t}\right) \wedge d t .
$$

While comparing the $(p+1, q-1,1)$ forms of $(2.4)$ both sides, we have

$$
0=\partial \omega^{\prime \prime} \text {. }
$$

Note. When $p=0$, ker $\partial^{p, q}=\Gamma_{a}\left(\Lambda^{0, q}(M)\right)$ and all $\omega^{\prime \prime \prime}$ in (2.2) vanish. When $q=0$, all $\omega^{\prime \prime}$ in (2.2) vanish.

3. A homotopy theorem for $\Gamma_{a}\left(\Lambda^{0 * *}(M)\right)$. Theorem (1.1) is an easy consequence of the following homotopy theorem.

(3.1) TheOREM. Let $g(z, t)$ be an analytic homotopy of holomorphic mappings $g^{0}$ and $g^{1}$ on a complex manifold $M$ (of $m$ complex dimensions), then the induced linear mappings $g^{0 *}$ and $g^{1 *}$ coincide on the cohomology groups of

$$
\begin{aligned}
& 0 \longrightarrow \Gamma_{a}\left(\Lambda^{0.0}(M)\right) \stackrel{\delta}{\longrightarrow} \cdots \stackrel{\delta}{\longrightarrow} \Gamma_{a}\left(\Lambda^{0 . q}(M)\right) \\
& \stackrel{\delta}{\longrightarrow} \Gamma_{a}\left(\Lambda^{0, q+1}(M)\right) \stackrel{\delta}{\longrightarrow} \cdots \stackrel{\delta}{\longrightarrow} \Gamma_{a}\left(\Lambda^{0, m}(M)\right) \longrightarrow 0 .
\end{aligned}
$$

Proof. If we can construct a chain homotopy

$$
H_{q}: \Gamma_{a}\left(\Lambda^{0 . q}(M)\right) \rightarrow \mathrm{\Gamma}_{a}\left(\Lambda^{0 . q-1}(M)\right)
$$

such that $\bar{\partial} H_{q}+H_{q+1} \bar{\partial}=g^{1 *}-g^{0 *}$, then the proof will be done.

The construction of $\left\{H_{q}\right\}$ proceeds as follows:

Let $\omega \in \operatorname{ker} \partial^{0 . q}(q \geqq 1)$ and $g^{*} \omega=\omega^{\prime}+\omega^{\prime \prime} \wedge d t$, where $\omega^{\prime}, \omega^{\prime \prime}$ are $(0, q, 0)$, $(0, q-1,0)$ forms in variables $(z, t)$ resp. Then $H_{q} \omega$ is defined to be

$$
\left.\left(H_{q} \omega\right)(z)=(-1)^{q-1} \int_{0}^{1} \omega\right)^{\prime \prime}(z, t) d t
$$


for $1 \leqq q \leqq m$ and $H_{q}=0$ for all other $q$. Clearly $H_{q} \omega$ is globally well defined and by (2.6), $H_{q} \omega \in \operatorname{ker} \partial$. To check that the $H_{q}$ form a chain homotopy, we have

$$
\partial H_{a} \omega=(-1)^{a-1} \int_{0}^{1} \partial \omega^{\prime \prime}(z, t) d t
$$

and by (2.4) and (2.5),

$$
H_{a+1} \partial \omega=(-1)^{a} \int_{0}^{1}\left(\partial \omega^{\prime \prime}+(-1)^{q} \frac{d \omega^{\prime}}{d t}\right) d t .
$$

Thus

$$
\bar{\partial} H_{q} \omega+H_{q+1} \partial \omega=\int_{0}^{1} \frac{d \omega^{\prime}}{d t} d t=\left.\omega^{\prime}\right|_{t=1}-\left.\omega^{\prime}\right|_{t=0}=g^{1 *}-g^{0 *} .
$$

4. Proof of Theorem (1.2). Let $p$ be fixed. The proof that the $\{i *\}$ are injective will be accomplished by the construction of a sequence of mappings $\lambda_{q}: \Gamma\left(\Lambda^{p, q}(M)\right) \rightarrow \operatorname{ker} \partial^{p, q}, q=0, \cdots, m$, and a chain homotopy $H_{q}: \operatorname{ker} \partial^{p, q \rightarrow \operatorname{ker}} \partial^{p, q-1}$ such that

$$
\bar{\partial} H_{q}+H_{a+1} \bar{\partial}=\mathrm{id}-\lambda i
$$

(i.e., $\lambda *^{*} i^{*}=\mathrm{id}$ ).

Let $g(z, t)$ be an analytic deformation of $M$ with $g(z, 1)=$ id and $g(z, 0)=$ point (notice that $g^{1 *}=$ id and whenever $(p, q) \neq(0,0), g^{0 *}=0$ ) and let $\omega$ be any $(p, q)$ form with $g^{*} \omega$ given by (2.2). Define mappings $\{\lambda\}$ by

$$
\left(\lambda_{q} \omega\right)(z)=(-1)^{p+q-1} \int_{0}^{1} \partial \omega^{\prime \prime \prime} d t
$$

for $0 \leqq q \leqq m$. For $\omega \in \operatorname{ker} \partial^{p, q}, H_{q}$ is defined by

$$
\left(H_{q} \omega\right)(z)=(-1)^{p+q-1} \int_{0}^{1} \omega^{\prime \prime} d t
$$

for $1 \leqq q \leqq m$ and $H_{q}=0$ for all other $q$. Clearly both $\lambda_{q}$ and $H_{q}$ are globally well defined, $H_{q} \omega \in \operatorname{ker} \partial^{p, q-1}$ as before, and $\lambda_{q} \omega \in \operatorname{ker} \partial^{p, q}$ is obvious. To check (4.1), we have, with the help of (2.4) and (2.5)

$$
H_{q+1} \bar{\partial} \omega=(-1)^{p+q} \int_{0}^{1}\left(\partial \omega^{\prime \prime \prime}+\bar{\partial} \omega^{\prime \prime}+(-1)^{p+q} \frac{d \omega^{\prime}}{d t}\right) d t .
$$

Thus

$$
\begin{aligned}
\left(\bar{\partial} H_{q}+H_{q+1} \bar{\partial}\right) \omega & =-\lambda \omega+\int_{0}^{1} \frac{d \omega^{\prime}}{d t} d t \\
& =-\lambda i \omega+\left(g^{1 *} \omega-g^{0 *} \omega\right)=\omega-\lambda i \omega .
\end{aligned}
$$


Note. When $p=0$, all $\omega^{\prime \prime \prime}$ vanish, thus $\lambda_{q} \omega=0$. Then from (4.1), it can be shown that with $M$ assumed in (1.2), Corollary (1.3) still holds for $M$.

\section{REFERENCES}

1. A. Aeppli, On the cohomology structure of Stein manifolds, Proc. Conf. Complex Analysis (Minneapolis, 1964), Springer, Berlin, 1965, pp. 58-70. MR 36 \#4588.

2. R. C. Gunning and H. Rossi, Analytic functions of several complex variables, Prentice-Hall, Englewood, Cliffs, N.J., 1965. MR 31 \#4927.

Department of Mathematics, State University of New York at Albany, Albany, New York 12203

Current address: Department of Mathematics, University of Montreal, Montreal, Quebec, Canada 